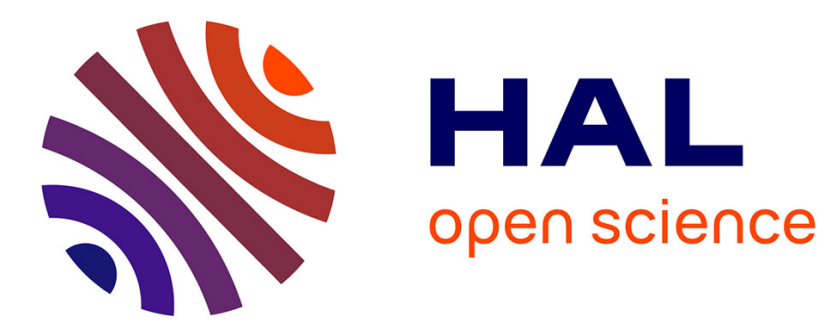

\title{
Multidomain Index Modulation for Vehicular and Railway Communications: A Survey
}

Ping Yang, Yue Xiao, Yong Liang Guan, Marco Di Renzo, Shaoqian Li, Lajos Hanzo

\section{- To cite this version:}

Ping Yang, Yue Xiao, Yong Liang Guan, Marco Di Renzo, Shaoqian Li, et al.. Multidomain Index Modulation for Vehicular and Railway Communications: A Survey. IEEE Vehicular Technology Magazine, 2018, 13 (3), pp.124 - 134. 10.1109/MVT.2018.2814023 . hal-01880304

\section{HAL Id: hal-01880304 https://hal.science/hal-01880304}

Submitted on 7 Jul 2020

HAL is a multi-disciplinary open access archive for the deposit and dissemination of scientific research documents, whether they are published or not. The documents may come from teaching and research institutions in France or abroad, or from public or private research centers.
L'archive ouverte pluridisciplinaire HAL, est destinée au dépôt et à la diffusion de documents scientifiques de niveau recherche, publiés ou non, émanant des établissements d'enseignement et de recherche français ou étrangers, des laboratoires publics ou privés. 


\title{
Multi-Domain Index Modulation for Vehicular and Railway Communications: A Survey
}

\author{
Ping Yang, Senior Member, IEEE, Yue Xiao, Member, IEEE, Yong Liang Guan, Member, IEEE, \\ Marco Di Renzo, Senior Member, IEEE, Shaoqian Li, Fellow, IEEE, and Lajos Hanzo, Fellow, IEEE
}

\begin{abstract}
Vehicular communications have recently attracted considerable attention due to a range of new applications and value-added features. Compared to conventional communication systems, vehicular communications suffer from more grave challenges due to the high-Doppler spread caused by the mobility of vehicles. In this context, index modulation (IM) constitutes a promising design alterative for vehicular communications due to its appealing benefits. A key feature of IM is that it conveys the information bits by using the specific indices of the transmit resources, such as antenna indices, subcarrier indices, precoder indices and channel-path indices. The resultant transmit signals tend to be sparse in one or several of the resource domains, which increases their robustness and reduces the detection complexity. In this article, we introduce the basic IM concept and highlight the applications of IM in the context of road vehicle-to-infrastructure (V2I), vehicle-to-vehicle (V2V), conventional railway and high-speed train communication systems. Finally, some design challenges of IM-based vehicular communications are listed for future studies.
\end{abstract}

Index Terms - High-speed train (HST), multipleinput multiple-output (MIMO), vehicular communications, index modulation (IM).

\section{INTRODUCTION}

A DVANCED vehicular systems have recently attracted a great deal of attention. High-speed train (HST) systems have been developed in many countries to connect major cities, which travel at the speed of $300 \mathrm{~km} / \mathrm{h}$. In 2016, some countries have started research on a new type of magnetic levitation based train that can reach $600 \mathrm{~km} / \mathrm{h}$, while the SpaceX company has initiated the 'Hyperloop' project aiming for supersonic speeds of up to $1200 \mathrm{~km} / \mathrm{h}$. Moreover, multiple companies, including Google and Volvo, have been testing self-driving cars on public roads for several years and recently the world's first public self driving taxi has been launched (http://www.bbc.com/news/business37181956). These new vehicular systems impose more

P. Yang, Y. Xiao, and S. Li are with the National Key Laboratory of Science and Technology on Communications, University of Electronic Science and Technology of China, 611731, Sichuan, China. (e-mail: yang.ping@uestc.edu.cn, xiaoyue@uestc.edu.cn, lsq@uestc.edu.cn).

Y. L. Guan is with the School of Electrical and Electronic Engineering, Nanyang Technological University, Singapore (e-mail: eylguan@ntu.edu.sg).

Marco Di Renzo is with the Laboratory of Signals and Systems (L2S), French National Center for Scientific Research (CNRS), University of Paris-Sud XI, 3 rue Joliot-Curie, 91192 Gif-sur-Yvette, France(e-mail: marco.direnzo@lss.supelec.fr).

L. Hanzo is with the School of Electronics and Computer Science, University of Southampton, Southampton SO17 1BJ, U.K. (email:lh@ecs.soton.ac.uk) stringent reliability requirements on the supporting communication services, in order to improve the safety and comfort. One of the most challenging tasks in this context is to conceive suitable physical-layer transmission technique [1].

For this research objective, previous study is much fruitful [1]. For example, the concepts of multi-antenna and multi-carrier techniques have been introduced into vehicular communications, such as the multiple-input multipleoutput (MIMO) and orthogonal frequency-division multiplexing (OFDM) schemes. On the one hand, these techniques have the potential of increasing the degrees of freedom with objective of tackling the highly dynamic nature of vehicular channels. More recently, in order to further improve the stringent reliability and rate requirements of vehicular communications, large-scale MIMO techniques have been introduced.

In general, the high-velocity vehicle-to-vehicle (V2V) scenarios are more challenging than the well-understood conventional fix-to-mobile (F2M) transmissions. With the increase of speed, the wireless MIMO vehicular channel becomes much more complicated, where the effect of the mutual electromagnetic coupling between different antenna elements and the spatio-temporal correlation [1] also has to be considered ${ }^{1}$. Hence, the conventional MIMO-aided or OFDM techniques suffer from more grave problem$\mathrm{s}$, such as the Doppler-induced inter-carrier interference (ICI), inter-antenna interference (IAI) and inter-antenna synchronization. Moreover, the road traffic and railway systems jointly form an integrated transportation network, where not only vehicle-to-infrastructure (V2I) and $\mathrm{V} 2 \mathrm{~V}$, but also train-to-train $(\mathrm{T} 2 \mathrm{~T})$, train-to-infrastructure $(\mathrm{T} 2 \mathrm{X})$, and train-to-vehicle (T2V) or even drone-to-drone and plane-to-plane communications will be included [1]. This makes the services in the vehicle network become more diverse and refined, in order to satisfy the safetyoriented and integrity-oriented demands.

For the sake of satisfying these demanding application scenarios and for innovatively exploiting the degrees of freedom of multiple antennas (space-domain) and multiple carriers (frequency domain), recently the family of index modulation (IM) techniques have been proposed [2], [3]. Specifically, IM relies on the generalized on/off keying principle applied to any of the available signal resource domains, in order to modulate the information bits on to the indices of the transmit resources. In general, the transmit signals of IM generated in the employed resource domains tend to be sparse. Moreover, IM techniques are

\footnotetext{
${ }^{1} \mathrm{It}$ is worth noting that the modeling of the MIMO vehicular channels is still a challenge, which is a vitally important research topic.
} 
capable of striking attractive tradeoffs amongst the range of conflicting system requirements, such as the rate-perchannel-use, the reliability, the hardware cost, the complexity and the energy efficiency, which provide potential opportunities to use them in vehicular communications.

This article aims for summarizing the latest research on IM transmission solutions for application to vehicular communications, which has recently gained attention. More important, another purpose of this article is to provide the scientific community with an overview of the most challenging aspects in the focused context of IM-based vehicular communication systems and to give useful hints about the way to tackle these challenges.

\section{Principle of Index Modulation}

\section{A. Basic Model}

Index modulation is a novel technique of transmitting information bits mapped to the indices of time-, frequency, and spatial transmit resources [3], in addition to the classic PSK/QAM constellations. The roots of IM can be traced back to the early frequency- or time-hopping techniques, which were recently extended to the spatial domain [2]. The general system model of IM is depicted in Fig. 1, where the information bit stream is first mapped to the joint bit vector. Then, this vector is partitioned into two sub-vectors, namely the index bits and the classic bits, which are used for selecting a unique transmit resource index (or indices) and mapped to a PSK/QAM symbol, respectively. Based on this process, the joint bit vector is ultimately mapped to an IM vector/matrix for transmission, as shown in Fig. 1.

Compared to classic modulation/transmission schemes, IM can be viewed as

- a new multi-dimensional hybrid modulation scheme, which exploits the indices of the transmit resources as additional dimensions, apart from the classic PSK/QAM.

- a novel irregular constellation design, whose constellation diagram constituted by the scaled versions of the complex-valued channel impulsive response taps. The resultant constellation tends to be irregular and continuous-valued.

More important, IM can be flexibly configured for diverse system configurations. As a result, it is found that some IM designs can be viewed as unified modulation/transmission frameworks, including many of the previously-developed transmission arrangements as their special cases. For example, the classic space time block code (STBC) and the vertical Bell labs layered spacetime (VBLAST) architectures constitute special cases of generalized space time shift keying (GSTSK) [3], where the indices of transmit matrices are employed for bit mapping. Moreover, IM can be jointly designed in combination with the classic transmission schemes in order to exploit their hybrid benefits.

Another important feature of IM is that the IM signals are often sparse in one or several resource domains, which can brings the following benefits:

- it can be exploited to reduce the transceiver cost including the detection complexity, since the actual number of activated transmit and receive responses is indeed reduced.

- it can reduce the destructive effects and be able to relax some stringent transmission requirements, i.e., ICI, IAI and the constraint of $N_{t} \leq N_{r}$ for spatial multiplexing.

Moreover, in addition to the above benefits, recent results have also shown that IM is capable of extending the link range and cell-radius, improving the reliability of communication, increasing the throughput of the network, whilst mitigating the multiuser interference [2]-[4]. These properties potentially lends the applications of IM in vehicular communications.

\section{B. Index Types and Examples}

As shown in Fig. 1-(2), we can exploit different degrees of freedom offered by the domains of time, frequency, antenna-index as well as, coding schemes and their hybrid combinations in the context of IM [2]-[5]. In these domains, there are various index types that can be used for conveying information. Some notable index types documented in the literature are: (a) the index of the transmit antennas; (b) the index of the receive antennas; (c) the index of the subcarriers; (d) the index of the code types; (e) the index of the channel impulsive response taps; (f) the index of the radio frequency (RF) mirrors; etc. Based on the specific resource domain used, we summarize these index types in Fig. 1. Accordingly, these options correspond to different IM designs, where types (a), (c) and (e) correspond to the well-known spatial modulation (SM), index-modulated OFDM (OFDM-IM) as well as to spatial and time-dispersion modulation (STdM) arrangements.

To be specific, in Fig. 1-(3) we present the design details of SM, OFDM-IM, STdM and hybrid IM schemes. As shown in Figs. 1-(3)-(a) and 1-(3)-(b), the SM/space shift keying (SSK), STdM and OFDM-IM rely on a similar principle, but follow different bit-to-symbol mapping approaches, which depend on the resource domains utilized. As a result, these IM techniques may perform differently in terms of their BER and detection complexity. To be specific, SSK relies exclusively on the transmit antenna indices to convey information, whilst dispensing with the classic PSK/QAM signaling, hence it imposes a lower complexity than SM and STdM. By contrast, in space-time aided IM, one out of the pre-defined space-time matrices was activated, rather than simply activating one out of the transmit antennas in order to disperse a PSK/QAM symbol, which is capable of achieving a beneficial diversity gain at the cost of a higher complexity imposed by the associated joint space-time detection. In general, these IM techniques have a similar equivalent system model, where the equivalent transmit vector includes only a single (or several) non-zero component(s). As a result, they may rely on similar detection algorithms, such as the singlestream based ML detector. More details concerning the comparisons of these IMs can be found in [2].

Moreover, it is worth noting that these index types can be combined for further improving the system's flexibility, as shown in Fig. 1-(3)-(d). Indeed, a specific degree of freedom can be achieved in different ways for an IM system by striking a compelling tradeoff among different 


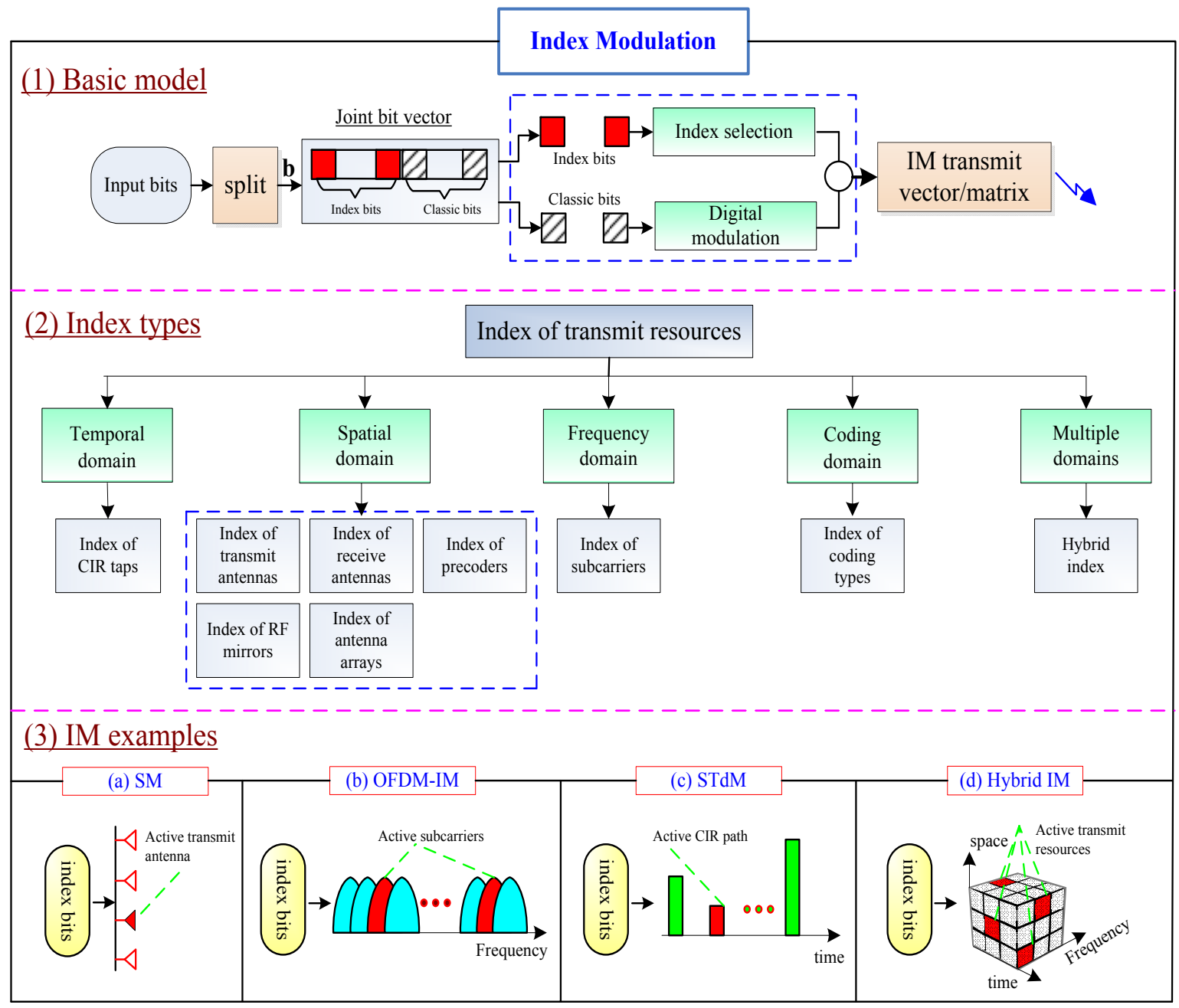

Fig. 1. Index modulation: the basic model, index types and some examples.

conflicting performance factors. For example, in multiantenna systems, in contrast to V-BLAST which has a high multiplexing gain, the family of SM-based schemes may offer only a logarithmic (rather than a linear) increase of the throughput with the number of transmit antennas. This limits the spectral efficiency of SM, but these flexible SM designs are capable of reducing both the number of transmit RF chains and the detection complexity. Recently, the development of IM has been leaned forward to its high-rate designs, as exemplified by the generalized SM and media-based modulation (MBM) scheme [5]. Specifically, generalized SM employs the combinations of antenna indices, while MBM relies on a number of RF mirrors near the transmit antennas, which are used to create different channel realizations based on the ON/OFF status of the mirrors. As shown in [5], MBM is capable of reducing the hardware cost, while maintaining a high throughput per channel use.

\section{INDEX Modulation fOR VEHICULAR COMmUnications}

Vehicular communication is emerging as a hot topic in both the academic and industrial research fields. The most unique feature in vehicular communications is the specific variety of services and its challenging propagation conditions. In vehicular communications, some particular factors, such as the vehicle traffic density, the spatial correlation and the high velocity of both the transmitter and/or the receiver, impose new challenges on the design of physical-layer transmission techniques.

As discussed in Section II, the flexibility and versatility of IM techniques facilitate their applications in vehicular communications, since they are capable of flexibly supporting diverse services and integrity requirements. More importantly, their robustness and reliability benefits can be readily exploited for combating the impairments imposed by the rapidly fluctuating vehicular channels.

In recent years, a flurry of research activities has been inspired by the design and the performance characterization of index modulation techniques conceived for different vehicular communication scenarios [6]-[13], such as the V2I, V2V, conventional railway and high-speed railway systems. Some potential problems routinely encountered in vehicular communications and their promising IMbased solutions are shown in Fig. 2. In general, for a specific vehicular scenario, different IM techniques ( and their combinations) exhibit different features and the appropriate IM technique has to be carefully selected depending on the specific system requirements. In this section, we review these promising advances in IM-based vehicular communications and discuss their potential benefits. 


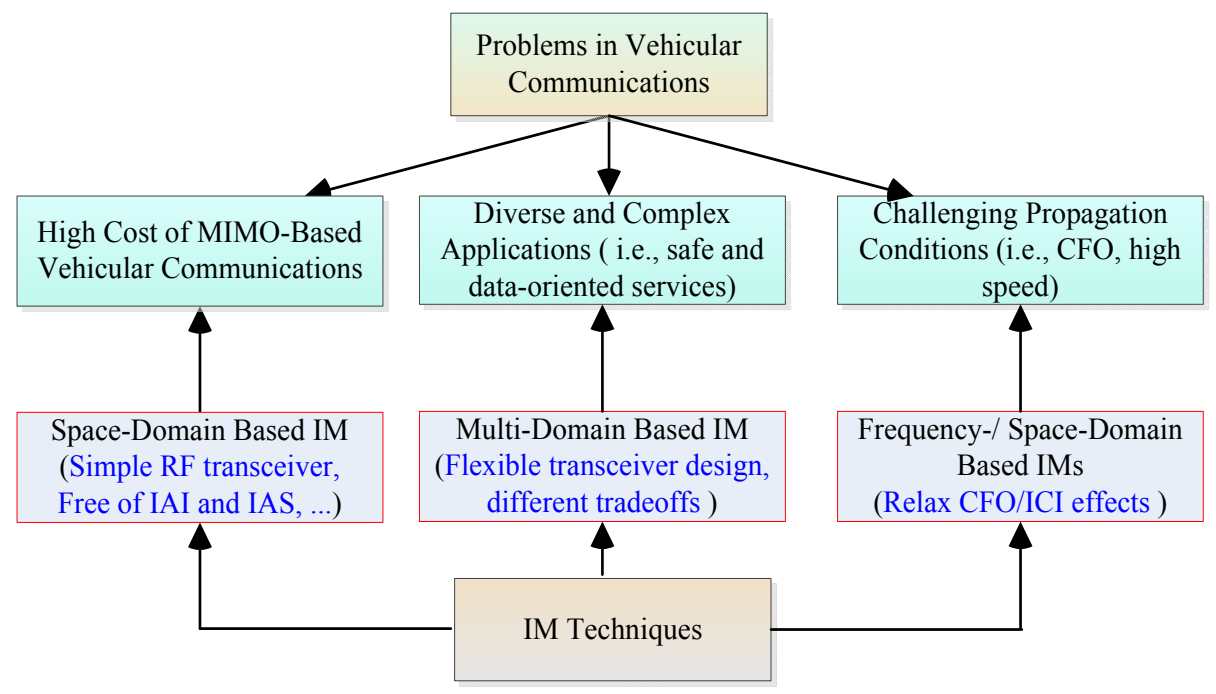

Fig. 2. Some potential problems in vehicular communications and their promising IM-based solutions.

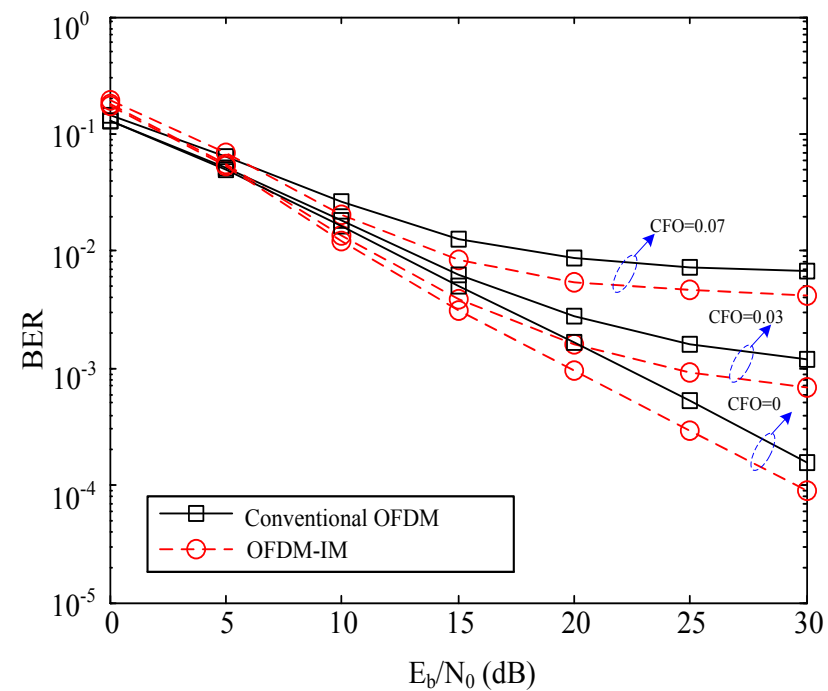

Fig. 3. BER comparison of conventional OFDM and OFDM-IM with interleaving under different CFOs in the Extended Vehicular A (EVA) channel.

\section{A. IM for V2V Communication}

$\mathrm{V} 2 \mathrm{~V}$ communication supports the wireless transmission of data between vehicles. One of the major goals of V2V communication is safety-related applications, such as the prevention of accidents. IM has been recommended as a promising solution for $\mathrm{V} 2 \mathrm{~V}[6],[7]$, since it is capable of improving the system performance, despite reducing the transceiver cost.

To be specific, in [6] a low-complexity version of SM (spatial-domain IM), namely SSK, was proposed for intervehicular communications, where a general V2V statistical channel model was employed for the system's performance analysis. In [6], the classic Rician, Rayleigh and doubleRayleigh channel models were also considered and closedform expressions of the uncoded and coded error probabilities were derived. Moreover, asymptotically tight upper bounds were derived for the average bit error probability and the simulation results demonstrated that SSK is capable of attaining an attractive performance in multiple scattering channels.
In contrast to the simple 2-D channel model of [6], a more realistic 3-D V2V MIMO vehicular channel model was employed for investigating the bit error ratio (BER) performance of SM [7], where the impact of vehicular traffic density, of the Doppler effect and of the channel's spatial correlation was jointly considered. It was shown in [7] that single-RF based SM strikes a better tradeoff in terms of throughput, complexity and cost, than the full$\mathrm{RF}$ chain aided conventional MIMO arrangements, such as STBC and V-BLAST.

\section{B. IM for V2I Communication}

V2I communication is concerned with the exchange of information between a vehicle and the infrastructure that may affect the vehicle, and vice versa. The primary objective of V2I is to improve road safety as well as to provide additional traveler information services. Apart from the dynamically fluctuating propagation and telltraffic, further challenges arise due to the asymmetric nature of the system, potentially reducing the robustness and reliability.

To tackle these issues, sophisticated IM techniques were proposed for V2I communication applications, by exploiting their appealing benefits. Specifically, in [8] a performance analysis framework was proposed for OFDM-IM (frequency-domain IM) operating in high-mobility multipath vehicular channels. It was shown in [8] that OFDMIM outperforms the classic OFDM and provides a signalto-noise ratio (SNR) gain of up to $9 \mathrm{~dB}$ at a speed of 100 $\mathrm{km} / \mathrm{h}$ for the specific setups considered.

In [9], a generalized version of OFDM-IM, namely OFDM-based quadrature IM (OFDM-QIM), was introduced for V2I communications. Compared to OFDMIM, OFDM-QIM independently employs the orthogonal in-phase and quadrature domains for index modulation and hence it becomes capable of doubling the system's throughput. It was found in [9] that OFDM-QIM outperforms the classic OFDM-IM scheme, when the appropriate carrier frequency offset (CFO) compensation method is adopted. Moreover, in [9] interleaving techniques were used for dispersing fading-induced burst of errors and a novel 


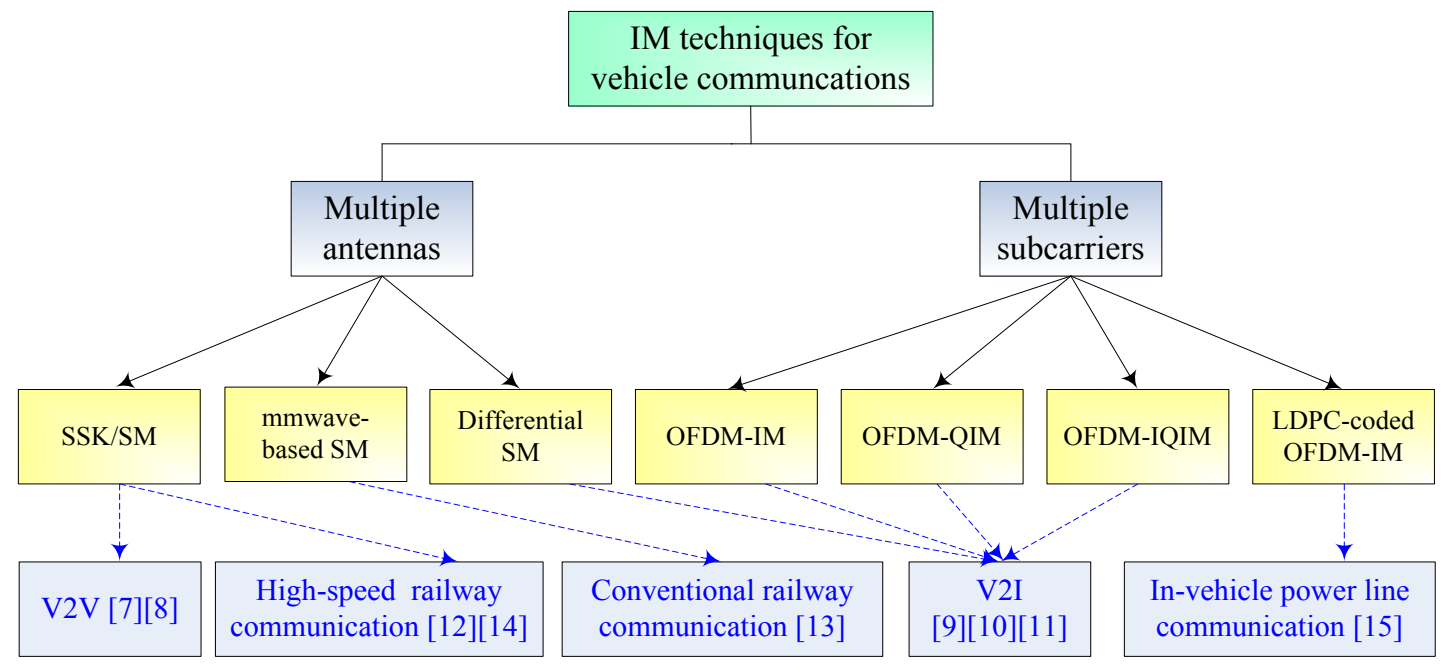

Fig. 4. IM techniques proposed for different vehicular communications.

OFDM-QIM combined with interleaved grouping (OFDMIQIM) was proposed. Specifically, similar to OFDM-QIM, the basic idea of OFDM-IQIM is to independently perform index modulation for both the in-phase and for the quadrature components of the symbol, whilst employing interleaved grouping to scatter the subcarriers of a subblock that were equally distributed across the OFDM block. As a result, compared to OFDM-QIM, OFDM-IQIM is capable of reducing the subcarrier correlation in each modulation unit and thus can improve the robustness against channel induced subcarrier correlation.

To exemplify the benefits of frequency-domain based IM, in Fig. 3, we compare the BER performance of OFDM and OFDM-IM combined with interleaving for different normalized CFOs in the classic Extended Vehicular A (EVA) channels. In Fig. 3, the simulation setups are as follows: the number of subcarriers is $N=1024$, the subcarrier separation is $15 \mathrm{kHz}$, the carrier frequency is $2 \times 10^{3}$ $\mathrm{MHz}$ and the normalized fractional CFOs are $0,0.03$ and 0.07 , respectively. In our simulations, the EVA channel model is considered, which represents a medium delay spread environment. Note that the EVA channel has 9 multipath components, which can be specified by their tap delays of $[0,30,150,310,370,710,1090,1730,2510] \times 10^{-9}$ (ns) and their relative powers of $[0,-1.5,-1.4,-3.6,-0.6$, $-9.1,-7.0,-12.0,-16.9](\mathrm{dB})$. It is observed in Fig. 3 that frequency-domain based IM (OFDM-IM) is more robust to CFO compared with conventional OFDM. This is due to the fact that some sub-carriers are inactivated in OFDMIM scheme, as illustrated in Fig. 1-(3)b.

Note that most of the above-mentioned schemes assume that perfect channel state information (CSI) is available at the receiver. However, it is challenging to acquire accurate CSI in high-speed vehicular systems. In order to dispense with CSI-estimation, the class of differential SM (DSM) was proposed in [10], where both point-to-point and amplify-and-forward aided cooperative systems were investigated in V2I channels. Theoretical and simulation results demonstrated that DSM is capable of operating within less than $3 \mathrm{~dB}$ compared to coherent $\mathrm{SM}$ at the same throughput, while requiring no CSI at the receiver, hence imposing a negligible pilot overhead.

\section{IM for Railway Communication}

Compared to the family of road traffic systems, light rail, subway, intercity railway, conventional and high-speed rail traffic systems are an alternative, more convenient and environmental-friendly way of allowing people to travel, which rely on sophisticated communication systems for supporting diverse applications and services, such as T2T, $\mathrm{T} 2 \mathrm{X}$ and T2V applications. Hence, their system capacity has to be improved to guarantee both high-rate Internet access for the passengers and high-reliability train-control signaling.

To this end, IM-based MIMO and massive MIMO schemes were also invoked for railway communication systems [11]-[13]. Specifically, in [11] SM was invoked for high-speed railway communications, where a nonstationary wideband vehicular channel model was considered and the associated space-time correlation function was invoked for characterizing the channel's nonstationary behavior. Moreover, in [11] the effects of realistic non-ideal channel estimation on the BER performance of MIMO channels for railway communications was investigated.

As a further advance, in [12] a hybrid SM-based beamforming scheme was proposed for railway communications in the mmWave band, where uniform planar arrays are equipped at the base station and uniform linear arrays are evenly deployed on the roof of the train. Specifically, in this hybrid scheme the conventional IM concept was used to select an active transmit antenna array and then the optimal RF beam was selected to transmit modulated symbols in the desired angular direction. It is shown in [12] that this hybrid analog-digital SM scheme strikes an attractive performance versus complexity tradeoff between the conventional SM and the full digital beamforming scheme.

More recently, in [13] a large-scale antenna aided IM scheme was proposed for high-speed railway communications, where the channel correlation imposed by the line of sight (LOS) propagation may erode the multiple antenna gain. Serendipitously, it was found that both the temporal and the spati-temporal correlation is actually mitigated by the high velocity of the train. 


\section{IM for Power-Line based In-Vehicle Communication}

IM has been extended to other advanced vehicular communication systems as well, such as power-line based in-vehicle communication. This novel technique uses the power lines inside the vehicle as a transmission medium and allows eliminating some of the wiring required for conveying data signals. To be specific, in [14] a LDPCcoded OFDM-IM scheme was proposed for in-vehicle power line communications, in order to combat the deleterious effects of dispersive channels and impulsive noise, while optimizing the energy efficiency.

In conclusion, we summarize the aforementioned IM applications for vehicular communications in Fig. 4.

\section{Further Design Issues}

As discussed in Sections II and III, the versatility of IM renders it a key enabler for vehicular communications. However, the research of this area is still in its infancy, hence there are numerous open issues to solve. This Section explores the main unresolved issues of IM designed for vehicular communications and provides to the reader with inspiration for tackling these issues.

\section{A. Performance Analysis in Broadband Fading Channels}

The wide-ranging studies disseminated in [6]-[14] have characterized some of the fundamental properties and benefits of IM techniques conceived for vehicular communications. For example, different analysis frameworks have been proposed for various IM schemes under different vehicular channel models, i.e., the second order scattering based propagation channels of [6] and the 3-D geometrybased stochastic channel model of [7]. However, these frameworks have been predominantly investigated in the context of non-dispersive narrow-band MIMO scenarios, whilst in practice most of the wireless channels exhibit frequency selective properties.

Recently, the IM designs have also been extended to the mmWave and visible light frequencies, where large bandwidths are available. However, the corresponding wideband channel models have to be considered in future research in the context of vehicular communication systems. A potential solution is to employ the momentgenerating function (MGF) for the associated analysis.

\section{B. Other High-Rate Designs}

Achieving a high reliability and high throughput is of salient importance in vehicular communications. However, as discussed in Section II and Fig. 1, the reliabilityversus-throughput tradeoff achieved by conventional IM techniques may be not optimal, which may not satisfy some specific requirements of vehicular communications.

To deal with this issue, several attractive increasedrate as well as energy-efficient IM techniques have been proposed. In detail, various generalized variants of SM and OFDM-IM schemes have been introduced, including the GSTSK, hybrid IM and MBM schemes of Section II. For SM design, the quadrature SM (QSM), the generalized SM (GSM) and their combinations (such as the generalized QSM (GQSM) scheme proposed in [15]) have been recently proposed for the sake of broadening the range of tradeoffs in terms of rate, complexity and RF costs, as shown in Fig. 5.

To be specific, as shown in Fig. 5, the conventional lowrate $\mathrm{SM}$ and SSK schemes have a low-cost single-RF-based transmitter, but they attain a low MIMO multiplexing gain. QSM relies on both the in-phase and quadrature dimensions to carry out index modulation and hence it improves the transmit rate of conventional SM, but it requires at least two RF chains and hence increases the dimensionality of the underlying detection problem. In contrast to QSM, GSM offers a linear increase of the transmit rate, but it requires more $\mathrm{RF}$ chains. The GQSM scheme of [15] is capable of further improving the spatial multiplexing gain by dividing the total number of the transmit antennas into sub-sets having two transmit antennas for each sub-set and then employing the QSM technique for each sub-set. Note however that its corresponding RF cost and complexity are increased, as depicted in Fig. 5 .

In Fig. 5, it is interesting to see that for $M=4$ the transmit rate of GQSM is identical to that achieved by spatial multiplexing. It is worth noting that this rate gain is achieved using fewer RF chains, namely $N_{t} / 2 \mathrm{RF}$ chains in GQSM versus $N_{t} \mathrm{RF}$ chains in spatial multiplexing. In other words, this is a $50 \%$ reduction in number of transmit $\mathrm{RF}$ chains in GQSM compared to VBLAST.

Moreover, besides itself evolution, IM can also combined with other high-rate techniques for further rate improvement.

\section{Detector Design}

As briefly noted before, the coherent versus noncoherent detection trade-offs also have to be documented, with a special emphasis on the high-Doppler vehicular scenarios. The fundamental problem is that every time the vehicular speed is doubled, the pilot overhead has to be doubled as well, hence imposing an excessive overhead. By contrast, differentially encoded and non-coherently detection systems impose an almost negligible overhead, but typically require $3 \mathrm{~dB}$ higher SNR. However, when they are compared under fair circumstances, assuming that the BER curves be appropriately shifted for the pilotaided coherent scheme to take into account the power assigned to the pilots, the non-coherent scheme might even outperform its coherent counterpart. Having said that, non-coherent schemes tend to develop an error-floor at high Doppler frequencies, which can be mitigated with the aid of joint multiple-symbol detection techniques relying on sphere-detection for reducing the complexity imposed by powerful but complex wide-window-based detection.

The performance of coherent schemes can also be improved with the aid of iterative joint channel estimation and data detection. This can also be combined with the powerful decision-directed channel estimation, where the receiver makes a hard-decision concerning those symbols, which are deemed to be reliable by the channel decoder. Provided that these symbols are indeed detected free from errors, they can be used in the successive joint channeland-data estimation iterations as pilots, since they are perfectly known.

Numerous future iterative receiver techniques can be used for improving the performance of IM systems, such as 


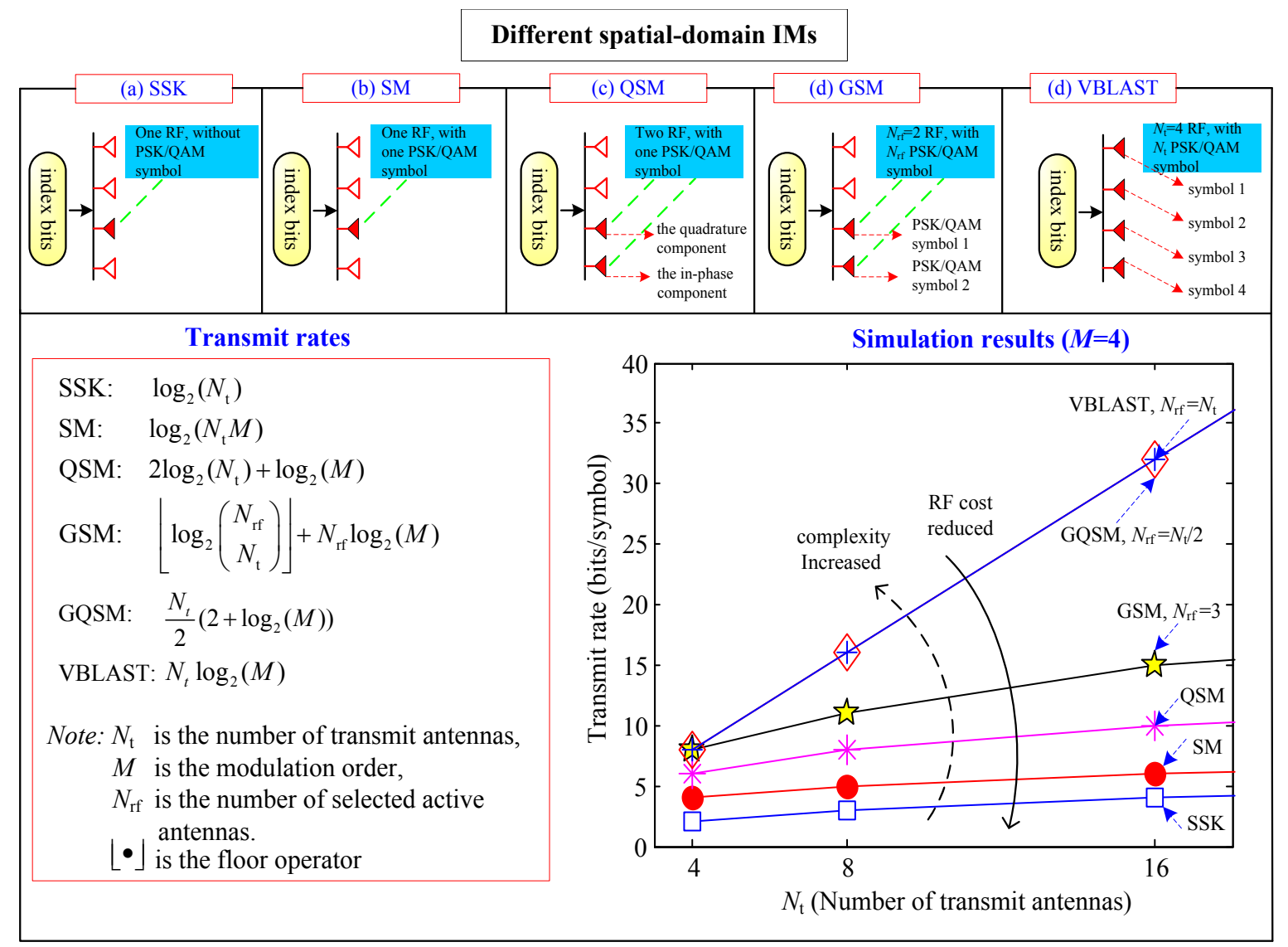

Fig. 5. The complexity and rate tradeoffs of different space-domain based IM schemes, where the modulation order is 4 .

turbo-synchronization and turbo-equalization. The specific choice of the most appropriate channel codes has to be based on Extrinsic Information Transfer Charts for the sake of achieving near-capacity operation. The employment of cooperative relaying also has to be studied along with appropriate relay-selection techniques. Furthermore, the design of IM-aided multi-user multi-cell systems is entirely open.

\section{Conclusions}

The research efforts invested in the design of IM have significantly increased in recent years motivated by its flexibility and versatility. Throughout this article we presented the main characteristics of IM techniques and their applications to vehicular communications. We commenced with the basic principle of IM and some specific IM designs. Then, we reviewed a range of recent research achievements related to IM designed for V2I, V2V, conventional railway and high-speed railway scenarios. Finally, we focused our attention on some future challenges in IM-based vehicular communication systems, which will hopefully serve as guidelines for their future development.

\section{ACKNOWLEDGMENTS}

The financial support of the National Science Foundation of China under Grant number 61501095, of the European Research Council's Advanced Fellow Grant and of the Royal Society's Wolfson Award and of International Science and Technology Cooperation Project of Sichuan Province(No.2017HH0009) are gratefully acknowledged.

\section{REFERENCES}

[1] C.-X. Wang et al., "Vehicle-to-vehicle channel modeling and measurements: Recent advances and future challenges," IEEE Commun. Mag., vol. 47, no. 11, pp. 96íC103, Nov. 2009.

[2] M. Di Renzo et al., "Spatial modulation for generalized MIMO: Challenges, opportunities and implementation," Proceedings of the IEEE, vol. 102, no. 1, pp. 56-103, Jan. 2014.

[3] E. Başar, "Index modulation techniques for $5 \mathrm{G}$ wireless networks," IEEE Commun. Mag., vol. 54, no. 1, pp. 168-175, Jul. 2016.

[4] M. Wen, et al., "Index modulated OFDM for underwater acoustic communications," IEEE Commun. Mag., vol. 54, no. 5, pp. 132-137, May 2016.

[5] Y. Naresh, et al., "On media-based modulation using RF mirrors," IEEE Trans. Veh. Technol., in press, 2016, DOI: 10.1109/TVT.2016.2620989

[6] K. P. Peppas, et al.,"Space shift keying transmission for intervehicular communications," IEEE Trans. Intell. Transp. Syst., in press, 2016, DOI: 10.1109/TITS.2016.2556082.

[7] Y. Fu et al., "BER performance of spatial modulation systems under 3-D V2V MIMO channel models," IEEE Trans. Veh. Technol., vol. 65, no. 7, pp. 5725-5730, July 2016.

[8] E. Başar, et al., "Orthogonal frequency division multiplexing with index modulation," IEEE Trans. Signal Processing, vol 61 , no. 22, Nov. 2013, pp. 5536-49

[9] M. Zhang, et al., "Quadrature index modulated OFDM with interleaved grouping for V2X communications," 2016 International Conference on Computing, Networking and Communications (ICNC), Kauai, HI, 2016, pp. 1-5.

[10] M. Zhang, et al., "Differential spatial modulation in V2X," 2015 9th European Conference on Antennas and Propagation (EuCAP), Lisbon, 2015, pp. 1-5.

[11] Y. Fu, et al., "Performance investigation of spatial modulation systems under non-stationary wideband high-speed train channel models," IEEE Trans. Wireless Commun., vol. 15, no. 9, pp. 6163-6174, Sept. 2016.

[12] Y. Cui, et al., "Hybrid spatial modulation beamforming for mmWave railway communication systems," IEEE Trans. Veh. Technol., in press, 2016, DOI: 10.1109/TVT.2016.2614005. 
[13] Y. Cui and X. Fang, "Performance analysis of massive spatial modulation MIMO in high-speed railway," IEEE Trans. Veh. Technol., vol. 65, no. 11, pp. 8925-8932, Nov. 2016.

[14] H. Zhang, et al., "LDPC-coded index-modulation aided OFDM for invehicle power line communications," 2016 IEEE 83rd Vehicular Technology Conference (VTC Spring), Nanjing, 2016, pp. 1-5.

[15] F. R. Castillo-Soria, J. Cortez-González, R. RamirezGutierrez, F. M. Maciel-Barboza, and L. Soriano-Equigua, "Generalized quadrature spatial modulation scheme using antenna grouping" ETRI Journal, vol. 39, no. 5, pp. 707-717, Oct. 2017. 